MIGUEL ARANGO MARÍN

FACULTAD DE DISEÑO INDUSTRIAL

UNIVERSIDAD PONTIFICIA BOLIVARIANA

MEDELLÍN, COLOMBIA

MIGUEL.ARANGO@UPB.EDU.CO
Fecha de recepción: 24/01/2021

Fecha de aceptación: 27/05/2021

Cómo citar: Arango Marín, M. (2021)

Diseño para las transiciones: una ruta formativa desde las identidades y los saberes artesanales. RChD: creación y pensamiento, 6(10), 01-17 DoI: $10.5354 / 0719-837 X .2021 .60871$

Revista Chilena de Diseño,

RChD: creación y pensamiento

Universidad de Chile

2021, 6(10)

http://rchd.uchile.cl

\section{Diseño para las transiciones: una ruta formativa desde las identidades y los saberes artesanales}

\author{
Transition Design: a Formative Route From \\ Identities and Artisan Knowledge
}

Resumen. En este trabajo proponemos una ruta formativa para aproximarnos a elucidar un Diseño para las transiciones, situado en las identidades y los procesos productivos locales. Esta propuesta se fundamenta al recabar formas de articulación entre el Diseño y algunos saberes artesanales del territorio colombiano, caracterizadas por unas lógicas de diálogo horizontal entre estudiantes de Diseño y grupos artesanales. El artículo se centrará en dar cuenta de nuestra apuesta formativa, por medio del análisis de tres momentos de nuestro ejercicio pedagógico: a) la preparación conceptual y metodológica para el trabajo entre el Diseño y la artesanía; b) el viaje, como metodología de aprendizaje y de afectación; c) el trabajo de Diseño participativo, como escenario para el aprendizaje desde la experiencia y el diálogo entre saberes. Haremos una revisión de tres experiencias de Diseño participativo desarrolladas con comunidades y asociaciones artesanales en los departamentos de Putumayo, Boyacá y Casanare, en Colombia, entre el 2015-2017. Como veremos, los resultados de estas articulaciones se han traducido en evidencias del proceso formativo de las y los estudiantes y en proyectos de Diseño orientados a preservar, potenciar y fortalecer los saberes artesanales de los grupos con las que se ha trabajado.

Palabras clave: Diseño para las transiciones, Diseño participativo, enseñanza-aprendizaje, saber artesanal, viaje como método

\begin{abstract}
In this paper, we propose a formative route to approach the elucidation of a transition Design located in the identities and local productive processes. This proposal is based on forms of articulation between Design and some artisanal knowledge of the Colombian territory, characterized by a horizontal dialogue between Design students and artisan groups. The article will focus on an account of our formative route through the analysis of three moments of our pedagogical exercise: a) the conceptual and methodological preparation for the work between Design and crafts; b) the journey as a learning methodology; c) participatory Design work, as a setting for learning from experience and dialogue between knowledge. We will review three participator Design experiences developed with artisan communities and associations in the departments of Putumayo, Boyacá and Casanare in Colombia, between 2015-2017. As we will see, the results of these articulations have translated into evidence of the students' formative process and in Design projects oriented to preserve, enhance and strengthen the artisanal knowledge of the groups with which we have worked.
\end{abstract}

Keywords: artisanal knowledge, participatory Design, teaching-learning, transition Design, travel as a method 
Figura 1. Esquema general de los tres momentos de la ruta formativa y las evidencias de aprendizaje que elaboran las y los estudiantes en cada uno de ellos (elaboración propia)

\begin{tabular}{|c|c|c|c|}
\hline $\begin{array}{l}\text { Momentos } \\
\text { de la ruta }\end{array}$ & $\begin{array}{l}\text { (i) Preparación } \\
\text { conceptual y } \\
\text { metodológica }\end{array}$ & $\begin{array}{l}\text { (i) El viaje como } \\
\text { método de aprendizaje }\end{array}$ & $\begin{array}{c}\text { (iii) Diálogos } \\
\text { desde el diseño } \\
\text { participativo }\end{array}$ \\
\hline $\begin{array}{l}\text { Resultados: } \\
\text { evidencias de } \\
\text { aprendizaje y } \\
\text { propuestas }\end{array}$ & $\begin{array}{l}\text { - Relatorías. } \\
\text { - Mapas conceptuales } \\
\text { de contextualización } \\
\text { del territorio. } \\
\text { - Categorías de } \\
\text { análisis: }\end{array}$ & $\begin{array}{l}\text { - Diarios de campo } \\
\text { - Herramientas de } \\
\text { recolección de } \\
\text { información } \\
\text { - Instrumentos de } \\
\text { análisis }\end{array}$ & $\begin{array}{l}\text { Propuestas de } \\
\text { diseño orientadas } \\
\text { preservar, potenciar } \\
\text { y fortalecer los } \\
\text { saberes artesanales }\end{array}$ \\
\hline
\end{tabular}

\section{Introducción}

En este trabajo proponemos una ruta formativa para aproximarnos a la elucidación de un Diseño para las transiciones situado en las identidades y los procesos productivos locales. Esta propuesta se fundamenta al recabar formas de articulación entre el Diseño y algunos saberes artesanales del territorio colombiano, caracterizadas por unas lógicas de diálogo horizontal entre estudiantes de Diseño y grupos artesanales. Dichos diálogos contribuyen a que el Diseño local encuentre, en parte, los cimientos necesarios para establecer su propio discurso. El camino que proponemos se despliega en la revisión crítica del ejercicio de enseñanza-aprendizaje que hemos adelantado en los últimos años en nuestra actividad como docentes de Diseño en el curso Módulo Producto e Identidad (MPI) de un pregrado en Diseño Industrial de una universidad privada en Medellín, Colombia y en la revisión de tres experiencias de Diseño participativo desarrolladas con comunidades y asociaciones artesanales en el Valle de Sibundoy en Putumayo, Ráquira en Boyacá y Hato Corozal en Casanare entre el 2015-2017.

Nuestra propuesta se centra en dar cuenta de la ruta de formación y de encuentro entre saberes, por medio del análisis de tres momentos de nuestro ejercicio pedagógico con sus respectivos resultados y evidencias: a) la preparación conceptual y personal para afrontar los retos propios de un trabajo de vinculación entre el Diseño y la artesanía; b) el viaje, como metodología de aprendizaje y de afectación, para la comprensión de la diversidad de las identidades culturales del territorio nacional; c) el trabajo de Diseño participativo, que en el caso de este artículo se expresa a partir de la vinculación con asociaciones artesanales de los tres lugares mencionados (ver Figura 1). Como veremos, es en este último momento donde se ponen en juego el entendimiento complejo de los saberes artesanales, las tensiones y contradicciones del diálogo entre el Diseño y la artesanía y, sobre todo, el potencial ético-social y vinculante del Diseño para las transiciones.

Cabe comentar además que seguimos aquí los planteamientos sobre las formas de enseñanza y pedagogía para la formación en Diseño expuestas por Miguel A. Ovalle (2005), donde se hace énfasis en el proceso de Diseño como un escenario para el aprendizaje con los otros: estudiantes, docentes y terceros involucrados. De este modo, se aprende en el colectivo y en permanente diálogo y relación integral con otras personas, espacios y objetos. El proceso de aprendizaje se da en un hacer y un pensar desde la construcción de un sentido de un proceso de Diseño situado. 
En efecto, encontramos que Oscar A. Fernández (2017) señala que el Diseño debe pensarse y enseñarse desde el contexto, las culturas y las realidades propias de cada país. Quien estudie Diseño hoy debe saber hacer cosas, y al mismo tiempo, saber por qué las hace. Para responder a ese por qué, vemos que el trabajo de Pía Lindemann, Carlos Amtmann y Gustavo Blanco (2015), ofrece unas ideas coherentes con nuestros propios planteamientos. Para estos autores, una de las claves está en propiciar espacios de aprendizaje basados en el reconocimiento directo con las personas y los entornos locales. A modo de síntesis, encontramos y suscribimos que es en la vinculación de los procesos de formación desde la experiencia, orientados a la comprensión de la heterogeneidad geográfica, cultural y social de los territorios, que se establecen unas condiciones ideales para una educación en Diseño más eficiente, efectiva y gratificante para las y los estudiantes.

En lo que sigue desplegaremos los aspectos conceptuales, metodológicos y experienciales que constituyen nuestra propuesta. En tal sentido, lo que presentaremos es una parte del proceso de sistematización y análisis crítico de lecturas, anotaciones, conversaciones, discusiones, viajes, procesos de Diseño participativo con grupos de artesanas y artesanos, y experiencias vividas con nuestros estudiantes en el transcurso de 10 años de docencia. Como parte clave de este ejercicio, y puesto que hablamos de una ruta de enseñanzaaprendizaje, es necesario hacer una síntesis de las evidencias del proceso formativo de las y los estudiantes que han transitado por el curso, como también de los proyectos de Diseño que han surgido del proceso de diálogo de las tres experiencias mencionadas. A partir de todo ello proponemos una vía para un quehacer del Diseño en transición desde y para lo local.

\section{Dimensión conceptual: ensanchamiento del diseño desde las transiciones y su diálogo con los saberes artesanales}

Para hablar del componente conceptual de nuestra ruta formativa hemos encontrado una interesante puerta de entrada en las ideas de Klaus Krippendorf (2016) sobre la necesidad de rediseñar el Diseño a partir de la constitución de un discurso disciplinar consistente. Según expone este autor, el discurso debe ser entendido como una forma potente del uso del lenguaje que estructura las disciplinas y que, en el caso del Diseño, ofrezca nuevas formas para el análisis social y cultural. De allí que plantee que el Diseño deberá encontrar su consistencia discursiva y práctica en aquello donde es particularmente fuerte: desde su interpretación de esos fenómenos socioculturales y desde su potencial de articulación a esos fenómenos a partir de su creación artefactual.

Entendemos con Krippendorf (2016) que una de las claves de su propuesta es construir, desde el Diseño, discursos que se relacionen más con las posibilidades de la interpretación social de las ciencias humanas y sociales que en el objetivismo de las ciencias naturales. Desde este punto de vista, el Diseño abre sus puertas de reflexión y de constitución de discursos posibles y de formas de hacer el Diseño articulados con procesos culturales y sociales concretos. Para traer estas reflexiones a nuestro contexto, somos conscientes como diseñadores y docentes que somos responsables de buscar formas de rediseñar nuestros propios discursos para encaminar nuestra práctica hacia sendas acordes con nuestras particularidades locales sustentadas en nuestro patrimonio territorial y en las multiplicidades identitarias y culturales. 
Si seguimos esa invitación de consolidación de un discurso que nos ayude a rediseñar el Diseño, encontramos ecos sugerentes en los planteamientos de Terry Irwin, Gideon Kossof y Cameron Tonkinwise (2015) con su propuesta del transition design o el Diseño para la transición. En términos generales, podemos decir que es una propuesta encaminada en restructurar las bases epistemológicas del Diseño para propender por un quehacer investigativo, de enseñanza y de proyectación del Diseño orientado a la realización de sociedades sustentables, situadas en lo local pero vinculadas con lo global, y que constituyan una relación simbiótica con el ecosistema en donde estén situadas. Para ello, es necesario que se consoliden nuevas formas de entender, transmitir, apropiar y llevar a la práctica los lineamientos del Diseño desde la transdisciplinariedad y el contacto directo con las personas que se verán afectadas con la actividad proyectual (Irwin, 2018).

Desde el ámbito latinoamericano, encontramos una estimulante apropiación de los planteamientos del Transition Design en Arturo Escobar (2016, 2017) cuando expone que es necesario iniciar una transformación de las disciplinas y los paradigmas en los que pensamos nuestra sociedad, nuestra economía, nuestra política y nuestra historia. De esta manera, dice Escobar, asistimos a un momento coyuntural atravesado por una profunda crisis medioambiental, política y social en el que requerimos apostar por discursos que no insistan en los modelos de desarrollo en clave de la individualización y el crecimiento económico neoliberales, sino más bien que se articulen con propuestas alternativas sustentadas en la solidaridad, el vínculo con el otro y en los significados de lo común.

Se trata de buscar la estructuración de discursos que posibiliten pensar en futuros posibles que apunten hacia transiciones realizables, no utópicas, de lo individual a lo colectivo, de lo puramente económico y rentable a lo cultural construido comunitariamente. Lo interesante de estas ideas es el señalamiento explícito de que, así como debe haber un discurso en transición, deberá haber un Diseño para las transiciones. Es precisamente en ese punto donde vemos las resonancias de la invitación hecha por Krippendorf (2016) de apuntalar un discurso propio desde el Diseño. Advertimos en ese Diseño centrado en las particularidades locales una posibilidad de abrir las puertas para la reflexión sobre las experiencias de vida de las personas, de las acciones a pequeña escala, del valor de las prácticas situadas y de la potencia en las iniciativas emergentes desde la articulación comunitaria.

Con ello, acogemos la invitación de Escobar $(2016,2017)$ de propender a un Diseño para las transiciones y unos diseñadores que encaminen su quehacer para participar en proyectos orientados a: a) apoyar la vida de las personas; b) el aprovechamiento de las cualidades locales; c) la promoción de los cambios sociales; d) un Diseño que converse, por igual, con lo experto y lo no experto a partir de nuevas prácticas disciplinares como el co-Diseño y el Diseño participativo.

Creemos que las claves para el ensanchamiento teórico y práctico en clave local del Diseño pueden estar en los posibles diálogos entre dicha disciplina creativa y los saberes artesanales. Es desde allí que se puede pensar, enseñar y hacer un Diseño para las transiciones, un Diseño que forme diseñadores 
que vean en los procesos sociales y culturales locales, no limitaciones sino potencialidades en las que pueda participar, no como experto sino como un actor social más que a través del accionar creativo active su potencial vinculante y facilite los necesarios procesos de comunicación y cohesión social (Montaña, 2010; Margolin \& Margolin, 2012; Ceballos, 2016). Llegamos así a una claridad en nuestra ruta: el Diseño en el que creemos es aquel que se expande en la forma en que se dice, en los modos en que se transmite y enseña, en la manera en que se practica y que se sustenta en los posibles diálogos que puede establecer con los saberes artesanales que enriquecen nuestra diversa geografía.

Antes de seguir hacia el componente metodológico de nuestro argumento, es momento de ofrecer nuestra perspectiva frente a dos nociones esenciales al reflexionar sobre apuestas de un Diseño engranado en procesos culturales en Colombia: la identidad y la artesanía. Sobre la primera, hemos visto lo poco provechoso que es aproximarse al problema de la identidad en búsqueda de lo esencial, lo puro y lo originario, puesto que este abordaje da cuenta de esencialismos conceptuales que encaminan la reflexión a los seductores, y profundamente problemáticos, mitos fundacionales de la raza y la pureza social.

Cuando pensamos y enseñamos sobre la identidad, preferimos referirnos a ella en plural, es decir, buscamos pasar de la idea de identidad a las identidades. Así, nuestro interés se encamina a la muticulturalidad y el carácter diverso (indígena, afro, árabe, mestizo, blanco) de un territorio como el nuestro, que pone en evidencia que las riquezas culturales nutren a nuestras identidades. Entendemos, con la ayuda de Arjun Appadurai (2001), Juan Diego Sanín (2008) y Néstor García Canclini (2009), que las identidades culturales, al vincularse con las dinámicas globales, entran en un proceso de tensión entre lo foráneo y lo local, entre las potentes narrativas del consumo contemporáneo y las tradiciones de sus territorios. De allí sigue que las identidades de los países latinoamericanos se puedan caracterizar por sus procesos de hibridación cultural, donde el agenciamiento social se hace patente en la medida que las prácticas creativas autóctonas no desaparecen en el escenario del libre mercado y la globalización homogeneizantes, sino que se resisten, se complejizan y se transforman permanentemente.

En relación a la segunda noción, hemos encontrado al menos cinco formas en las que se aborda la artesanía: aquella que la considera un saber premoderno, ya superado, que va a tender a desaparecer (Juez, 2002); otra que la considera como un saber valioso que le puede otorgar valor a los procesos de Diseño (Quiñones, 2003; Molina, 2009; Solórzano, 2011); una tercera que busca reivindicar su potencial inmaterial y educativo, para descentrar la atención de su carácter preponderante de mercancía (Amaya, 2009; Vega, 2012); una cuarta y estimulante forma de abordarla como una manifestación de las resistencias culturales, sociales y creativas de comunidades indígenas, que puede tener resonancia más general en otras comunidades artesanales (Barrera, 2015); una quinta y sugestiva forma de comprender la artesanía, que la reivindica como un saber valioso en sí mismo que se basta en lo manual y lo cotidiano (Grisales, 2015, 2017). 
A partir de la consideración de estas perspectivas sobre las identidades y la artesanía, hemos consolidado la postura establecida desde el MPI que concibe los saberes artesanales como formas de manifestación culturales que son vigentes, que han resistido y resisten procesos modernizantes del mercado y de la globalización. Entendemos que esas resistencias se han dado desde las comunidades, de allí que reconozcamos que la artesanía se ha bastado y se basta a sí misma. Por ello, nos alejamos de un Diseño rescatista, paternalista e instrumentalista de los saberes artesanales y las personas que los hacen posible. Reconocemos además el gran valor que reposa en los saberes artesanales, en sus procesos de manufactura manuales, sus ritmos cotidianos de producción, su estrecha vinculación con tradiciones y conocimientos pasados y su inherente articulación con la geografía y las materias primas locales.

\section{El viaje como método de enseñanza-aprendizaje de un diseño situado en lo local}

Como lo hace explícito el profesor Germán Ferro (2009, 2010, 2011), el viaje es un espacio para el desprendimiento de los prejuicios, para el enriquecimiento espiritual y para la transformación profunda de quien lo emprende. Viajar es el método, es la búsqueda, es la iniciación, es enfrentarse a otras localidades, es un ejercicio de comprensión, es un espacio donde el conversar con el otro se constituye como instrumento clave para los procesos de enseñanza y de formación. En el viaje es necesario dejarse contagiar y afectar por el otro que no conozco, valorar su experiencia, sus saberes y la versión del mundo que tiene. Si esto se logra, la propia realidad se amplía y los horizontes de pensamiento se ven claramente enriquecidos.

Es en este contexto, donde la experiencia del viaje se consolida como método, que desde el MPI hemos realizado viajes hacia territorios, donde hemos contrastado nuestra primera aproximación conceptual con lo vivencial, con el contacto y diálogo de las personas locales. Es claro que para realizar estas salidas no basta con una aproximación conceptual como la que buscamos sintetizar en el apartado anterior, más si seguimos los señalamientos de Ferro y tenemos en cuenta las significativas implicaciones personales y en la sensibilidad de que estos desplazamientos implican.

En este sentido, preparar el viaje es para nosotros una etapa coyuntural de la ruta que queremos ofrecer en estas páginas. No solo es el espacio en donde se pone en discusión con los estudiantes la propuesta conceptual; además se constituye como el momento en el que buscamos aproximarnos al territorio, los saberes artesanales, las particularidades culturales e identitarias y los aspectos generales de los municipios o corregimientos que visitaremos.

Una parte de especial relevancia de este proceso es cuando conseguimos definir cuáles serán las preguntas que procuraremos responder en esos territorios y a partir de allí definimos unas herramientas para el levantamiento de esa información. La constante de nuestro proceso es enmarcar nuestra indagación en el ámbito de la investigación cualitativa y, por lo general, hacemos uso de las herramientas y las estrategias propias de la antropología, en particular del método etnográfico. Entendemos con Eduardo Restrepo (2016) que la etnografía ofrece hoy en día un batería de herramientas y formas de proceder que están 
disponibles para disciplinas más allá de la antropología y la sociología. De allí que hagamos algunos préstamos de dichas herramientas para recolección de información tales como: la observación participativa y no participativa, las entrevistas diversas y el diario de campo.

Como parte clave de esos préstamos, nos valemos de la amplia experiencia de la antropología para reforzar algunos aspectos relevantes del tipo de viaje que se requiere hacer dentro de la ruta del MPI. Por ejemplo, encontramos a Rosana Guber (2001) quien nos ofrece una máxima sencilla de escribir y de enunciar, pero bastante compleja de comprender y practicar: quien está en un proceso de investigación etnográfica debe luchar permanentemente con sus prejuicios y procurar, en lo posible, entender al otro en sus propios términos.

En nuestra experiencia, hemos visto como cada viaje, cada lugar y cada encuentro proponen formas de relacionamiento particulares y formas distintas de aplicar y emprender nuestra propuesta de ruta formativa de Diseño. Por estos motivos, en el próximo apartado veremos con cierto detalle tres viajes de especial significado para nuestro propósito.

\section{La ruta de formativa en diseño vista desde tres experiencias}

De todos los viajes que hemos emprendido ha habido tres que se han constituido como especialmente significativos. Nos referimos en particular a las experiencias vividas en el Valle de Sibundoy en Putumayo, en el municipio alfarero de Ráquira en Boyacá y en el municipio de Hato Corozal en Casanare. Decimos que son significativas ya que en la revisión que hacemos de ellos encontramos la validación de la ruta formativa que proponemos. En lo que sigue presentaremos cada una de las experiencias desde una doble dimensión: a) una esquemática, que da cuenta de la aplicación de la ruta formativa en cada viaje realizado para hacer explícitos algunos datos relevantes como la cantidad de estudiantes que han participado y los resultados de cada uno de los tres momentos de la ruta, traducidos en evidencias y propuestas particulares de Diseño; b) otra narrativa, que se apuntala en la primera, y que busca englobar nuestra propuesta desde unos relatos de lo vivido.

La ruta formativa en el Valle de Sibundoy, Putumayo (2015-2016) Las experiencias que tuvimos en el Valle del Sibundoy en el Alto Putumayo a través de dos visitas de una semana en el 2015 y en el 2016, fueron posibles gracias al contacto previo con un programa de formación superior que tiene la universidad donde trabajamos, en particular con una de las estudiantes de dicho programa. Luego de varias conversaciones con María Concepción Juajibioy, o Conchita como le gusta que la llamen, líder Kamëntsá y hermana Inga, logramos coordinar una visita a su territorio. A partir de la puesta en juego de nuestra ruta, nos permitió aproximarnos y reconocer con ella, su familia y su comunidad, sus prácticas cotidianas, sus costumbres, sus saberes artesanales y medicinales, y también sus proyectos educativos y comunitarios (ver Figura 2).

Los Inga y los Kamëntsá son dos grupos étnicos que han padecido y resistido diferentes embates de carácter colonial, primero por los procesos de desplazamiento ocasionados por la Conquista en el siglo XVI, luego por procesos de evangelización católica de los siglos XIX y XX, hasta la búsqueda de expoliación reciente de sus territorios para la explotación minera a 
Características generales del grupo de estudiantes

Cantidad de estudiantes

Cantidad de grupos y

proyectos realizados
32

10

$\begin{array}{ll}\begin{array}{l}\text { Momentos } \\ \text { de la ruta }\end{array} & \begin{array}{c}\text { (i) Preparación } \\ \text { conceptual y } \\ \text { metodológica }\end{array}\end{array}$

(i) El viaje como
método de aprendizaje

(iii) Diálogos

desde el diseño participativo

- Relatorías: 10 relatorías realizadas por los grupos de estudiantes.

- Mapas de contextualización del territorio: 10 mapas

Resultados: evidencias de aprendizaje $y$ propuestas conceptuales que se construyeron de forma colectiva.

- Categorías de análisis: cada uno de los 10 mapas dieron pie para consolidar unas categorías de investigación y de análisis.
- Diarios de campo: 32 diarios de campo registraron la experiencia del viaje. Herramientas de recolección de información: diseño y aplicación de entrevistas, guías de toma de fotografías y cartografías.

- Instrumentos de análisis: realización de 10 infografías discutidas en sesiones de trabajo en la salida de campo.

- Relatorías: giraron en torno de la problematización de las relaciones del diseño y las manifestaciones artesanales de las comunidades indígenas.

- Mapas de contextualización del territorio: posibilitaron una primera aproximación a la riqueza cultural, identitaria y social de las comunidades Inga y Kamëntsá.

- Categorías de análisis: en consonancia con el contexto, algunas de ellas: la cosmogonía, el saber artesanal, la enseñanza de la tradición y la gastronomía local.

- Diarios de campo: la mayor parte de los diarios centraron su atención en describir las prácticas cotidianas, las creencias religiosas, el uso de las lenguas inga y kamëntsá y los ingentes esfuerzos de la comunidad por preservar sus tradiciones.

Consideraciones generales de los resultados
- Herramientas de recolección de información: se aplicaron, sobre todo, en espacios de reunión comunitario con el apoyo y acompañamiento de las personas que hicieron las veces de anfitrionas.

- Instrumentos de análisis: se compilaron y analizaron en los infográficos en clave de categorías orientadas hacia la comprensión del patrimonio cultural de las comunidades Inga y Kamëntsá.

- Propuestas de diseño: 8 de los 10 proyectos fueron entregados a la comunidad para su validación, puesta en consideración y uso de las propuestas que estimarán de su interés y utilidad. A grandes rasgos, los proyectos se encaminaron al apoyo de la transmisión del conocimiento hacia las y los niños de ambas comunidades, al fomento de recuperación de las chagras y a la valoración de su sistema de creencias y de organización comunitaria.
Figura 2. Esquema de la ruta formativa aplicada a la experiencia del Valle de Sibundoy, Putumayo (elaboración propia) gran escala (Barrera, 2015). En voz de mujeres y hombres Inga y Kamëntsá, nos enteramos que a estos grandes procesos de aculturación se le suman problemas económicos, políticos y sociales a una escala de lo cotidiano, caracterizados por las condiciones de precariedad material de muchas familias de la comunidad, por el poco reconocimiento del valor de la voz política de ambos grupos étnicos, por la pérdida paulatina de su lengua y por la ausencia de relevo generacional para preservar sus tradiciones culturales. 


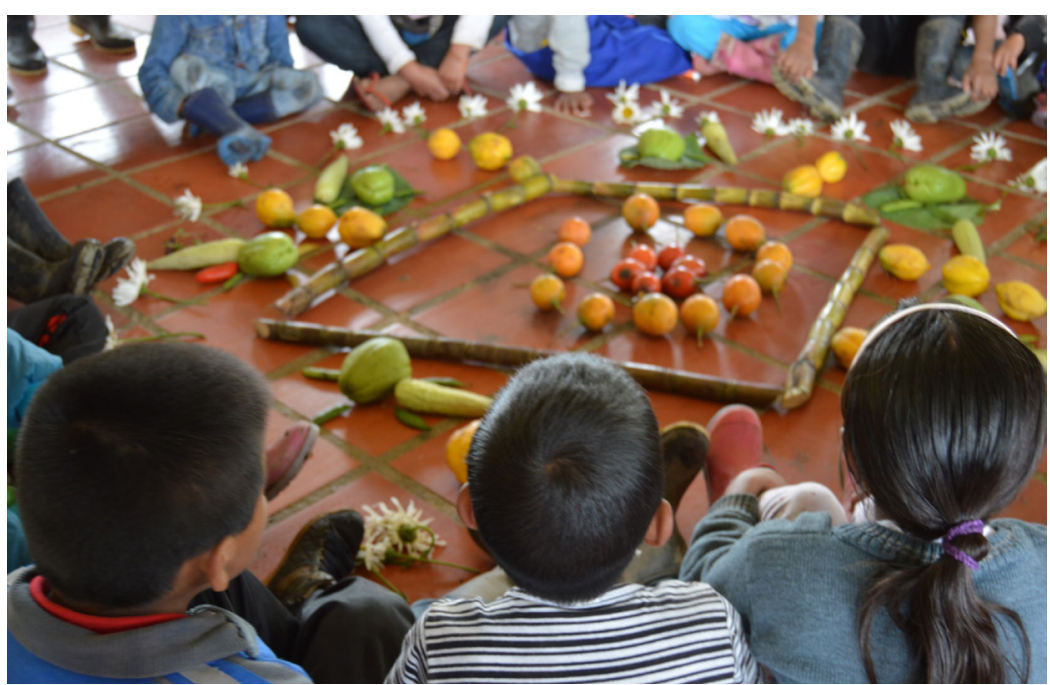

A pesar de todas estas presiones centenarias y de ese contexto adverso, fue sugerente para el grupo de estudiantes y docentes del MPI ser partícipes de diversas reuniones comunitarias y visitas a taitas, mamitas, maestros y maestras artesanas, así como también encuentros con los representantes del poder político de la comunidad. Decimos que resultó sugerente, pues gracias a esos espacios de diálogo atento encontramos sólidas iniciativas desde la actividad cotidiana de las mujeres y hombres de ambas etnias para re-construir sus tradiciones culturales y, a partir de ellas, asentarse en su presente y construir su futuro. Así lo pudimos ver a través de: a) la forma como utilizaban dignamente su lengua; b) el respeto que profesaban por sus taitas y su conocimiento de las plantas y sus ancestros; c) la manera en que sus saberes artesanales se materializan en objetos tejidos y tallados para su venta y, sobre todo, para dar cuenta que existen en ese territorio; d) los procesos en que buscan retornar a sus prácticas de agricultura doméstica con sus chagras; e) los proyectos educativos encaminados a que niños y jóvenes de la comunidad conozcan su lenguaje, sus tradiciones y sus narraciones en sus propios términos (ver Figura 3).

Ahora bien, nuestro interés de vivir y presentar estas experiencias no era un asunto puramente constatativo y de formación académica, pues sabíamos que nuestro papel en la comunidad debía rendir sus frutos como diseñadores. Con la información compartida se realizaron propuestas materiales concretas para apoyar los procesos educativos y de resistencias culturales que hemos expuesto. Es aquí donde emerge el componente participativo de estas experiencias en el Valle de Sibundoy: luego de la recolección de la información, el equipo del MPI realizó una serie de propuestas desde el Diseño que se materializaron y retornaron al territorio para ser usadas y luego evaluadas por la comunidad, para señalar en qué puntos habían sido acertadas, en que otros no y cómo sería posible mejorarlas.

Como ejemplo de este ejercicio, cabe retomar el proyecto de un grupo de estudiantes que captaron con especial claridad lo relevante de poner el quehacer del Diseño al servicio del rico patrimonio cultural de las personas
Figura 3. Niños y niñas de la comunidad Inga y Kamëntsá en un encuentro educativo en torno a su cosmovisión y el estrecho vínculo de la tierra y sus cultivos (fotografía tomada en 2016 por el equipo del MPI en el Valle del Sibundoy, Putumayo, Colombia) 
Figura 4. Proyecto de Diseño realizado por un grupo de estudiantes del MPI luego del viaje al Valle del Sibundoy (fotografía tomada en el 2016 por el equipo del MPI en Medellín, Antioquia, Colombia)

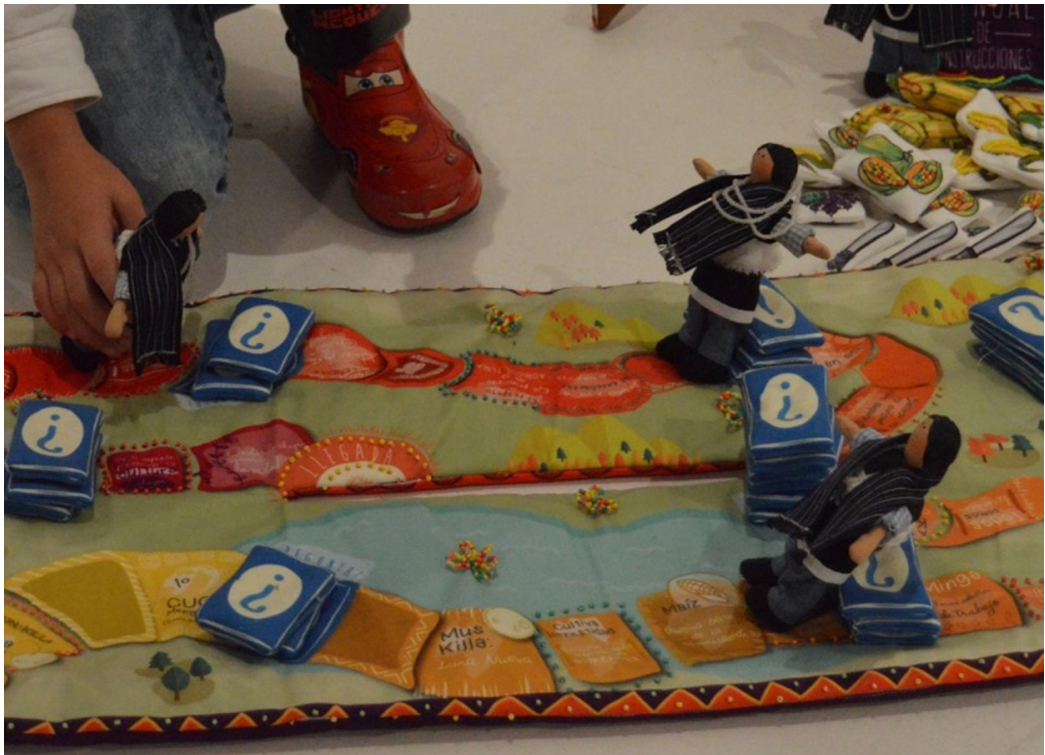

que nos habían acogido. Su proyecto, compuesto por un tablero de juego y unos pequeños muñecos de tela, que acogían como referencia la fisionomía, los objetos y la indumentaria de los Inga y los Kamëntsá, tuvo como propósito fundamental servir de mediador en los procesos de recuperación y puesta en valor de la lengua y las costumbres de la comunidad por medio de la enseñanza a niños y niñas de ambos grupos étnicos (ver Figura 4).

Lo significativo de este ejemplo tiene que ver con la forma en que el Diseño puede volcar su esfuerzo creativo y sus formas de materialización y producción a procesos de resistencia cultural encaminados a la vinculación comunitaria, y, al mismo tiempo, con el descentramiento de la atención del diseñador, que deja de lado su protagonismo y se engrana, como un componente más, a las dinámicas sociales con las que busca vincularse. Gracias a los procesos de validación, encontramos que el proyecto había sido integrado de tal manera a las lógicas de la comunidad que incluso fue replicado en otros grupos con los recursos técnicos con los que contaban los gestores culturales encargados de su divulgación.

\section{La ruta formativa en Ráquira, Boyacá, 2017}

Nuestra visita al municipio de Ráquira en Boyacá se realizó por un período de una semana en febrero del 2017. Para este viaje, aprovechamos nuevamente algunos puentes que se habían establecido con una asociación de artesanas y artesanos de la zona denominada Ráquira Tours. Este municipio alfarero fue por mucho tiempo un lugar de nuestro interés, ya que conocíamos la tradición centenaria de trabajo con el barro que ha pervivido en este territorio, en buena medida gracias los procesos de materialización artesanales vigentes en la zona. Dicho interés fue constatado, y rápidamente ampliado y complejizado, al iniciar y aplicar los tres momentos de nuestra propuesta formativa en el trabajo con la asociación (ver Figura 5). 
Ruta formativa aplicada a las experiencias de Ráquira, Boyacá (2017)

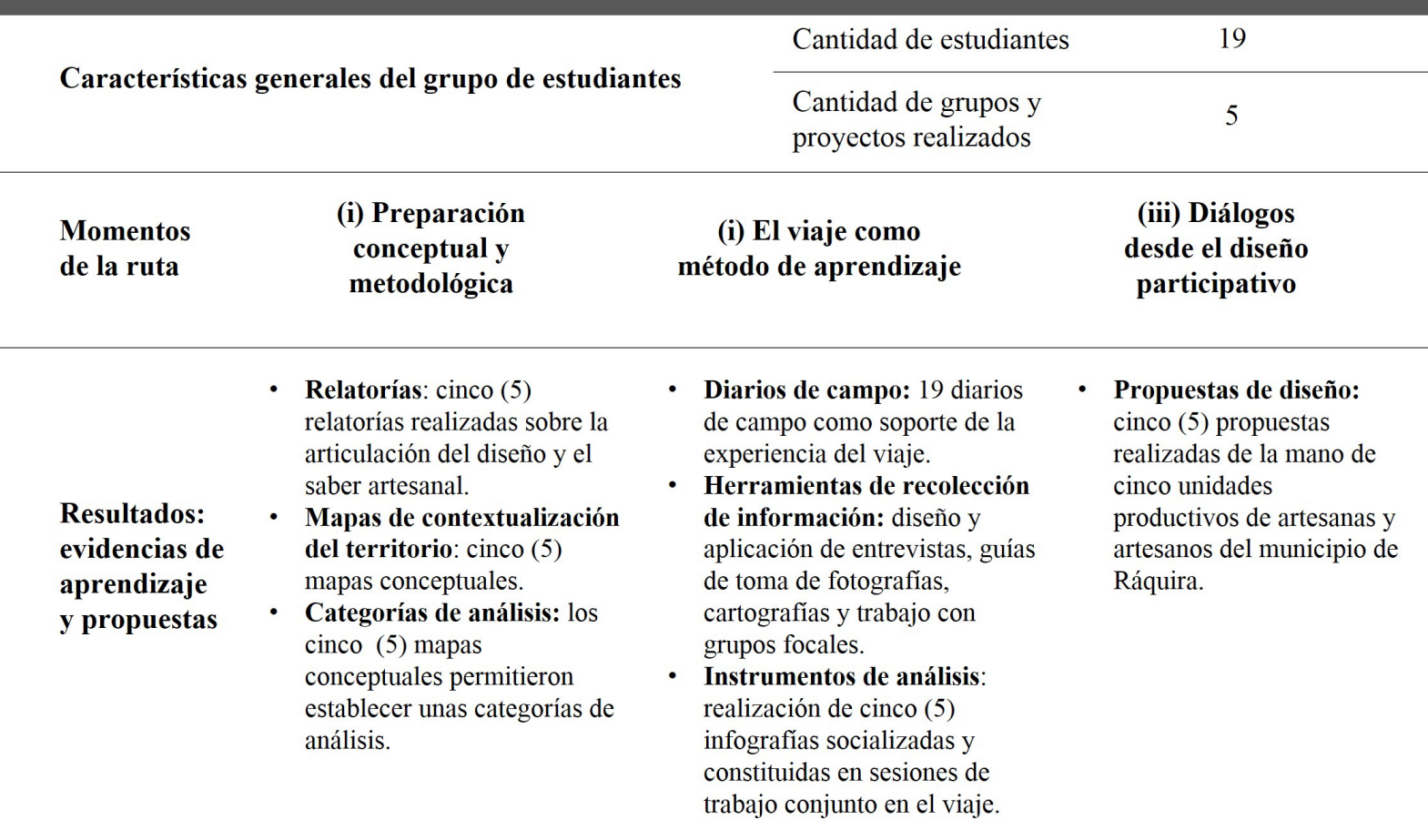

- Relatorías: tuvieron un particular énfasis en el oficio ceramista y la estrecha vinculación de este con el territorio y las materias primas relacionadas con el oficio.

- Mapas de contextualización del territorio: se elaboraron en clave de la comprensión de las características sociales, culturales e históricas del territorio boyacense.

- Categorías de análisis: giraron en torno a la tradición ceramista, al legado ancestral del territorio y al fortalecimiento asociativo.

\section{Consideraciones generales de los resultados}

- Diarios de campo: registraron la innegable presencia del saber ceramista y sus posibles fortalezas como colectivo, la consideración de la fauna, la flora y el paisaje como fuentes de inspiración y las permanencias en el barro de una cosmovisión indígena que pobló esos territorios.

- Herramientas de recolección de información: se aplicaron en su mayoría en los espacios de socialización y encuentro con las y los artesanos.

- Instrumentos de análisis: las infografías explicitaron que las claves de la propuesta de diseño deberían ser la valoración de la tradición ancestral, la diferenciación desde las particularidades del entorno y de cada taller y el fortalecimiento asociativo.

- Propuestas de diseño: todas las propuestas se desarrollaron desde un trabajo participativo en donde estudiantes y artesanos establecieron una relación de diálogo y materializaron unas propuestas encaminadas a la diversificación y diferenciación de sus productos frente a los demás talleres. Los resultados del ejercicio se socializaron en voz del grupo de artesanos y estudiantes y quedaron como parte de la memoria de cada taller para ser replicados.
En el marco del panorama rico y complejo ofrecido por nuestra ruta, pudimos percatarnos, entre otras cosas, de la vigencia de la cerámica en este municipio. Ciertamente, la vivacidad del oficio ceramista en Ráquira se manifiesta con claridad en su zona rural, que alberga más de 300 talleres que trabajan el barro con técnicas de conformación artesanales y semi-industriales (ver Figura 6).
Figura 5. Esquema de la ruta formativa aplicada a la experiencia de Ráquira, Boyacá (elaboración propia) 
Figura 6. Fábrica de producción de cerámica semi-industrial (fotografía tomada en el 2017 por el equipo del MPI en Ráquira, Boyacá, Colombia)

Figura 7. Grupo de estudiantes del MPI trabaja de la mano con uno de los artesanos del proyecto en su taller (fotografía tomada en el 2017 por el equipo del MPI en Ráquira, Boyacá, Colombia)
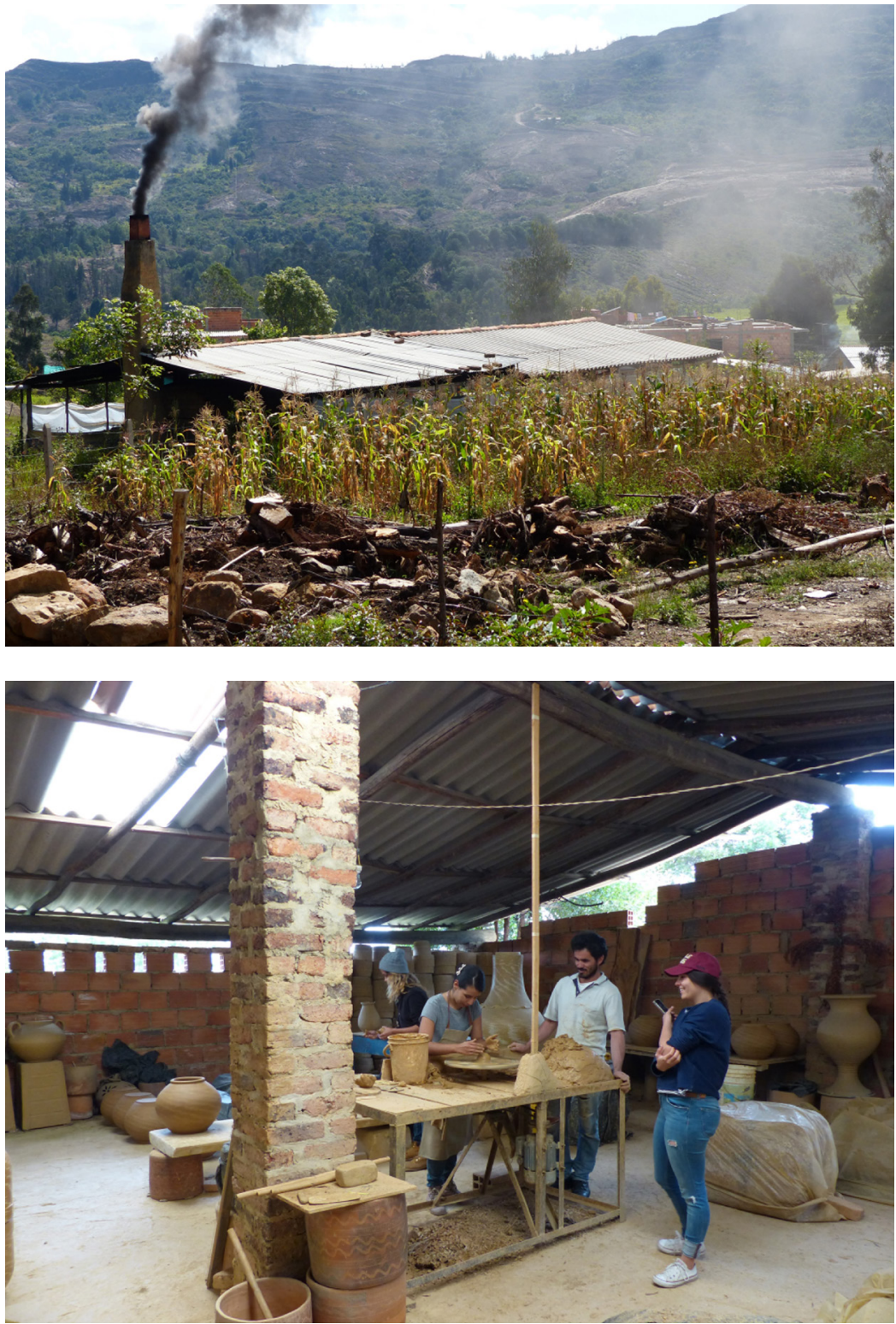

El ejercicio que realizamos incorporó varias estrategias y metodologías de Diseño. Consistió en diseñar o rediseñar un producto de carácter comercial que oxigenara la oferta que tenía cada taller de la asociación. En cuatro días se estudiaron las demandas de los clientes habituales y las fortalezas productivas del taller y su lenguaje, se conceptualizó e ideó de manera colaborativa entre artesanos y diseñadores en formación y se materializó gracias a la maestría artesanal de los miembros de la asociación (ver Figura 7). Este ejercicio correspondió a las metodologías de Diseño participativo. La finalidad era el desarrollo de productos serializados y su posterior comercialización a través de canales de distribución tradicionales (almacenes y ferias). 
Ruta formativa aplicada a las experiencias de Hato Corozal, Casanare (2017)

\section{Características generales del grupo de estudiantes}

Cantidad de estudiantes

12

Cantidad de grupos y

proyectos realizados

\section{5}

\begin{tabular}{|c|c|c|}
\hline $\begin{array}{l}\text { Momentos } \\
\text { de la ruta }\end{array}$ & $\begin{array}{l}\text { (i) Preparación } \\
\text { conceptual y } \\
\text { metodológica }\end{array}$ & $\begin{array}{l}\text { (i) El viaje como } \\
\text { método de aprendizaje }\end{array}$ \\
\hline
\end{tabular}

- Relatorías: cinco (5) relatorías elaboradas sobre la relación entre el diseño, la artesanía y las manualidades. Mapas de contextualización

Resultados: evidencias de aprendizaje y propuestas del territorio: cinco (5) mapas conceptuales desarrollados colectivamente.

- Categorías de análisis: de los mapas emergieron unas categorías de indagación y análisis para la comprensión del territorio llanero.
- Diarios de campo: 12 diarios de campo que compilaron la experiencia de las y los estudiantes durante el viaje.

Herramientas de recolección de información: diseño y aplicación de entrevistas, guías de toma de fotografias $\mathrm{y}$ entrevistas con grupos focales.

- Instrumentos de análisis: realización de cinco (5) infografias construidas en colectivo.
- Propuestas de diseño: cinco (5) propuestas de diseño traducidas en cinco (5) cartillas para promover las potencialidades creativas de cada una de las mujeres con las que se realizó un ejercicio de diseño participativo.

- Relatorías: giraron alrededor de los límites conceptuales y los posibles encuentros entre las manualidades y los saberes artesanales.

- Mapas de contextualización del territorio: procuraron dar cuenta de las disputas territoriales, los saberes artesanales y la impronta de las mujeres en el pie de monte llanero colombiano.

- Categorías de análisis: se orientaron a reflexionar sobre las transformaciones territoriales, la relación entre la industria y la ruralidad, las manifestaciones culturales y la gastronomía.

- Diarios de campo: dieron cuenta del radical cambio geográfico de un escenario andino a otro de un horizonte plano y sin montañas. Asimismo, se pusieron en evidencia la riqueza musical y

\section{Consideraciones generales de los resultados} gastronómica y el nivel de relevancia de la mujer llanera en las labores cotidianas y de creación.

- Herramientas de recolección de información: fueron parte integral de la mayor parte de los encuentros con la asociación de artesanas.

- Instrumentos de análisis: con su elaboración se reforzó la idea de la relevancia de las labores cotidianas de la mujer, su potencial creativo y la relevancia de su saber y su capacidad asociativa.

- Propuestas de diseño: las cartillas se quedaron en la asociación con el interés de que fueran un material de consulta e hicieran las veces de memoria del proceso. Se elaboraron como propuestas puntuales que apoyaran, en la mayoría de los casos, los procesos creativos y de ideación centrados en la riqueza territorial de la zona. Todo lo cual buscaba el fortalecimiento asociativo, la generación de espacios de formación, encuentro y socialización de las mujeres que componían la asociación.

Los resultados de este proceso fueron cinco propuestas encaminadas a cumplir un doble propósito: a) establecer elementos diferenciadores de las propuestas artesanales de cada taller a partir de los intereses, expectativas y tradiciones relevantes de las y los artesanos; b) propender al fortalecimiento de la asociación para explicitar que una de sus fortalezas radicaba en el trabajo colectivo. En torno al primer propósito, hubo propuestas significativas porque centraron su atención en potenciar la experticia artesanal del taller en
Figura 8. Esquema de la ruta formativa aplicada a la experiencia de Hato Corozal, Casanare (elaboración propia) 
Figura 9. Grupo de estudiantes del MPI observa el trabajo de una artesana de la asociación (fotografía tomada en el 2017 por el equipo del MPI en Hato Corozal, Casanare, Colombia)

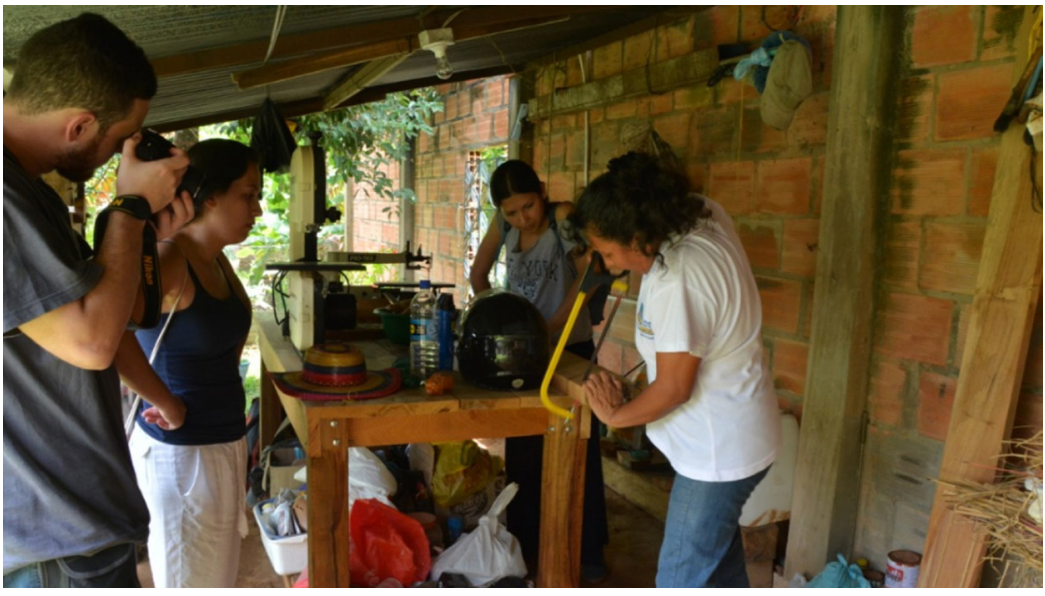

el cual trabajaron y buscaron articularla con la sensibilidad propia del grupo de estudiantes. Por ejemplo, hubo una propuesta que hizo abstracción del paisaje cercano, ya naturalizado por los artesanos, para elaborar piezas cerámicas con morfologías y texturas completamente novedosas y sustentadas en el entorno. En cuanto al segundo propósito, resultó especialmente revelador, ya que los docentes y estudiantes del MPI comprendimos que, por un momento, nuestro papel fue articularnos a las lógicas propias de la asociación y servir, por medio de la puesta en práctica del Diseño, como un incentivo para fortalecer una iniciativa que ellos ya habían iniciado.

\section{La ruta formativa en Hato Corozal, Casanare, 2017}

La experiencia en Hato Corozal, que se llevó a cabo durante una semana en agosto del 2017, inicia unos años antes debido a un ejercicio de asesoría a la Asociación de Artesanos de Hato Corozal en el que varias mujeres (algunas de ellas de linaje artesanal de talla de totumo o elaboración de cotizas, otras con la habilidad manual para tejer, bordar y pintar), realizaban productos que comercializaban en su municipio y en algunas ferias esporádicas en Yopal. Luego de haber tenido unos procesos de formación para el desarrollo de productos y un fortalecimiento vocacional de años, estas mujeres deciden aceptar la invitación de realizar un proyecto con el MPI para, a partir de la aplicación de nuestra propuesta formativa, buscar con ellas el desarrollo de nuevos productos que hablaran de su territorio y de lo que ellas entendían como su identidad llanera (ver Figura 8).

En el trabajo directo con la asociación fue evidente para nosotros que, a diferencia de las y los artesanos de Ráquira, en Hato Corozal muchas de las mujeres apenas estaban aprendiendo algunas técnicas de tejido y de pintura. El proceso que emprendimos se caracterizó, a través de la conversación y la realización de talleres participativos, por un diagnóstico de las unidades productivas que hacían parte de la asociación, sobre sus potencialidades y posibilidades productivas (ver Figura 9).

Gracias a esta actividad comprendimos que esta asociación no requería del Diseño de nuevos productos, sino un apoyo a partir de la sistematización, compilación y divulgación de estrategias para el fortalecimiento y los procesos de creación de los oficios que algunas mujeres estaban 


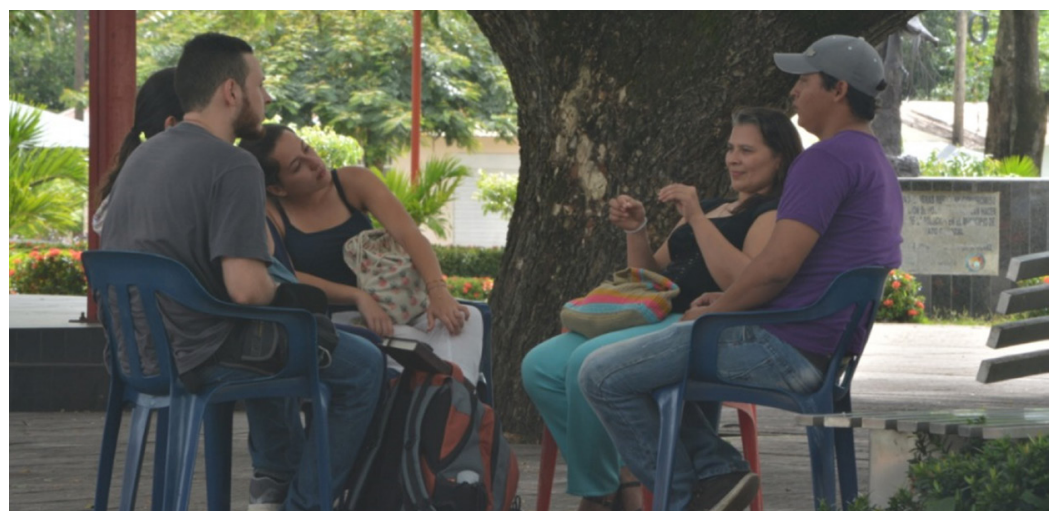

aprendiendo (ver Figura 10). Todo esto, que se realizó de forma participativa entre el grupo del MPI y las artesanas, se materializó en unas cartillas que compilaron todo el proceso y que fueron elaboradas a mano por el grupo de estudiantes con materiales disponibles en el municipio.

Nuestra participación se sustentó en el apoyo de estas mujeres para potenciar el proceso de organización y gestión de la asociación. A este complejo reto se sumó una variable de la que nos hicimos consientes con rapidez gracias a la personalidad de algunas de las integrantes: la agrupación se constituía para ellas en un espacio de autonomía femenina y de toma de decisiones como colectivo de mujeres. Como en otros proyectos que hemos realizado, se hizo explícita para nosotros una constante de nuestro trabajo: el papel preponderante de la mujer como garante de los saberes artesanales. Este hecho, que fue el común denominador de las tres experiencias que aquí hemos narrado, pone en evidencia que los procesos de asociatividad femenina han aportado y aportan cotidianamente a la construcción de las memorias, a la transmisión de conocimientos y al cuidado de sus comunidades.

A modo de síntesis, las tres experiencias descritas ponen de manifiesto que la ruta formativa que proponemos propende a que el proceso de aprendizaje de las y los estudiantes de Diseño se dé desde el reconocimiento de la heterogeneidad geográfica, étnica, cultural y social de los diversos territorios que componen una nación pluriétnica y multicultural como la colombiana. Es una ruta que le apuesta a un proceso de formación desde la experiencia, para generar condiciones de aprendizaje contextualizadas y encaminadas a la interiorización y apropiación de las competencias por parte de las y los estudiantes.

Las evidencias de aprendizaje presentadas en los tres momentos de la ruta nos lo constatan. Lo han demostrado la coherencia, el nivel de apropiación y la calidad de buena parte de las relatorías, los mapas, los instrumentos de recolección y análisis, los diarios de campo y las propuestas que han resultado de los diálogos desde el Diseño participativo. Reconocemos, sin embargo, que nuestra propuesta ha tenido sus agujeros, sus desaciertos y retos por resolver. Por un lado, algunos estudiantes (una minoría) han transitado por el curso sin compenetrarse con sus contenidos. Por otra parte, ha sido difícil compaginar los tiempos y expectativas académicas con las lógicas de las asociaciones y comunidades artesanales con las que hemos trabajo. Esto último ha complejizado la continuidad en los procesos y 
el seguimiento para evaluar los ecos que han tenido los ejercicios de Diseño participativo realizados, luego de que pasa el tiempo.

Dentro de las lógicas de trabajo del MPI es una tradición, al regresar del viaje y en la parte final del curso, abrir un espacio para la retroalimentación general de lo vivido, de los asuntos a valorar y de aquellos que deben ser ajustados. Sobre todo, para que las y los estudiantes expresen qué ha significado para ellos vincularse en un proceso formativo como el que este curso propone. En todos los años que hemos acompañado este proceso, y en particular en las tres experiencias que hemos compartido en estas páginas, la constante es que se hable de lo vivido en el MPI como un momento provechoso y significativo para el proceso formativo en otras maneras de ver $y$ hacer desde el Diseño. En palabras de un estudiante que estuvo en el proceso de Diseño participativo en el pequeño municipio llanero de Hato Corozal: “(...) lo que más me impactó de esta experiencia, fue que al estar allá trabajando con esas mujeres, me di cuenta de que en acontecimientos pequeños y que involucran pocas personas pueden pasar cosas realmente potentes y significativas" (Tomás Flórez, comunicación personal, 24 de agosto del 2017).

\section{A modo de cierre}

Creemos que hemos cumplido con nuestro propósito de presentar, desde tres experiencias, la ruta formativa en Diseño que por años hemos andado y reflexionado. Hemos buscado aproximarnos a modos de hacer que compaginan con nuestras ideas y nos ofrecen posibilidades de llevar a la práctica nuestro quehacer como diseñadores en clave de la comprensión de las particularidades culturales e identitarias que sitúan los saberes artesanales y enriquecen nuestro territorio.

Como hemos tratado de mostrar, nuestra propuesta se asienta en la enseñanza de una forma de entender el Diseño; una forma de pensarlo, decirlo y hacerlo. Un Diseño que busca encontrar su sentido en un discurso que apunte a su rediseño y que se logre enunciar desde y para las transiciones sociales, culturales, políticas y ambientales. Un Diseño que apunte a otros mundos posibles que abran alternativas al desarrollo corrosivo del híperconsumo. Mundos posibles que, si bien sabemos son minoritarios, somos conscientes de que ya existen, en iniciativas comunitarias, en colectivos sociales y políticos, en los procesos de resistencia étnicos y culturales, en la ruralidad y en lo urbano.

Gracias a las experiencias de los viajes realizados y las comunidades con las que hemos estado en contacto, nos hemos hecho conscientes de que la disciplina que enseñamos posee un potencial vinculante significativo que encuentra especial resonancia en las apuestas de construcción de lo colectivo. Estas experiencias nos ha posibilitado mirar en nuestros viajes asuntos que se nos hubieran pasado por alto y que se han constituido como esenciales en la puesta en práctica de dicho potencial vinculante del Diseño para las transiciones: los procesos de resistencia culturales, la recuperación del lenguaje, la valoración del potencial educativo de los saberes artesanales, la reivindicación de los tiempos y resultados del trabajo manual artesanal, la relación armónica que ciertas comunidades establecen con su entorno, la comprensión de lo potente que se encierra en acciones menores, y las búsquedas por la autonomía de mujeres y hombres que ven en sus posibilidades locales unas vías para hacerlo. La práctica del Diseño, creemos, puede aportar a esos procesos. 


\section{References}

Amaya, H. (2009). Cultura, artesanía y desarrollo. Mas D, (5), 25-36.

Appadurai, A. (2001). La modernidad desbordada. Ediciones Trilce S.A.

Barrera, G. (2015). Autonomía artesanal. Creaciones y resistencias del pueblo kamsá. Pontificia Universidad Javeriana.

Canclini, N. G. (2009). Culturas híbridas. Grijalbo.

Ceballos, M. (2016). Diseño industrial expandido como categoría aplicable e innovadora de la Gestión Cultural [Tesis de maestría no publicada]. Universidad de Antioquia.

Escobar, A. (2016). Autonomía y diseño. La realización de lo comunal. Universidad del Cauca.

Escobar, A. (2017). Diseño para las transiciones. Etnografías contemporáneas, 3(4), 32-63. http:// revistasacademicas.unsam.edu.ar/index.php/ etnocontemp/article/view/428/1074

Fernández, O. A. (2018). La pedagogía del diseño. ArDin. Arte, Diseño e Ingeniería, 7, 14-40. https://doi. org/10.20868/ardin.2018.7.3757

Ferro, G. (2009). Guías de observación y valoración cultural. Apuntes, 22(1), 34-53.

Ferro, G. (2010). Guía de observación etnográfica y valoración cultural: santuarios y oficios. Apuntes, 23(1), 56-69.

Ferro, G. (2011). Guía de observación etnográfica y valoración cultural: fiestas y semana santa. Apuntes, 24(2), 222-241.

Grisales, A. (2015). Artesanía, artes y diseño. Una indagación filosófica acerca de la vida cotidiana y el saber práctico. Universidad de Caldas.

Grisales, A. (2017). El olvido de la cotidianidad. Artesanía, arte y territorio. Universidad de Caldas.

Guber, R. (2001). La etnografía, método, campo y reflexividad. Norma.

Irwin, T. (2018). The Emerging Transition Design Approach. En Design Research Society [DRS] 2018: Catalyst, Volume 3 (pp.968-989). https://doi:10.21606/dma.2017.210

Irwin, T., Kossoff, G. \& Tonkinwise, C. (2015). Transition Design Provocation. Design Philosophy Papers, 13(1), 3-11. https://doi.org/10.1080/14487136.2015.1085688

Juez, F. M. (2002). Contribuciones para una antropología del diseño. Gedisa.
Krippendorf, K. (2016). Rediseñar el diseño: una invitación a un futuro responsable. Infolio, 5, 1-21. http://infolio.es/ articulos/krippendorff/krippendorfo5.htm

Lindemann, P., Amtmann, C. \& Blanco, G. (2015). Evaluación de la formación profesional de estudiantes de diseño industrial en base a experiencias en el ámbito rural. Estudios Pedagógicos, 16(1), 125-141. https://doi.org/10.4067/S0718-07052015000100008.

Margolin, V. \& Margolin, S. (2012). Un modelo social de diseño: cuestiones de práctica e investigación. Revista Kepes, 9(8), 61-71. http://190.15.17.25/kepes/downloads/Revista8_4.pdf

Molina, L. (2009). Diseño y tendencias del sector artesanal. Mas D, (5), 37-46.

Montaña, J. (2010). Diseño asociativo: del diseñador autor al diseñador facilitador. En A. M. Gracía (Comp.), Diseño, artesanía e identidad. Experiencias académicas locales de Diseño Artesanal en Colombia y El Salvador (pp. 23-48). Axis Mundi Institución Universitaria Colegio Mayor del Cauca.

Ovalle, M. A. (2005). Constructivismo en la pedagogía del diseño industrial: ¿Qué aprenden los alumnos? Revista de Estudios Sociales, 21, 37-52. https://doi.org/10.7440/res21.2005.03.

Quiñones, A. C. (2003). Artesanía y diseño en Colombia. En E. A. Quiñones (Comp.), Reflexiones en torno a la artesanía y el diseño en Colombia (pp. 1-26). Pontificia Universidad Javeriana.

Restrepo, E. (2016). Etnografía: alcances, técnicas y éticas. Envion Editores, Departamento de Estudios Culturales, Pontificia Universidad Javeriana.

Sanín, J. D. (2008). Estéticas del consumo. Configuraciones de la cultura material. Universidad Pontificia Bolivariana.

Solórzano, A. (2011, 8 de septiembre). Estudio sociohistórico sobre la iconografía de la cerámica utilitaria del Carmen del Viboral en Antioquia [ponencia]. Design Activism and Social Change Conference, Barcelona, España. http://www.historiadeldisseny.org/ congres/pdf/28\%2oSolorzano,\%2oAugusto.pdf

Vega, D. (2012). El aprendizaje de la artesanía y su reproducción social en Colombia. Educación y territorio, 2(1), 89-ı12. https://www.jdc.edu.co/ revistas/index.php/reyte/article/view/415 